\title{
Acquiring direction from new contacts
}

DOI:

$10.1038 / \mathrm{nrm} 2244$

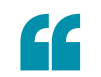

...cell

adhesion can

regulate the

establishment

of cell polarity.
In many cell types, proteins are asymmetrically distributed over the cell surface, which creates separate apical and basolateral membrane domains that uniquely contribute to cell and tissue function. Formation of the basolateral membrane domain has been observed to coincide with a second cellular process - E-cadherin-mediated formation of cell-cell contacts. Nejsum and Nelson now show that these two events are mechanistically linked, suggesting that changes in cell adhesion can regulate the establishment of cell polarity.

The authors studied the basolateral membrane protein aquaporin-3 (AQP3) and the closely related

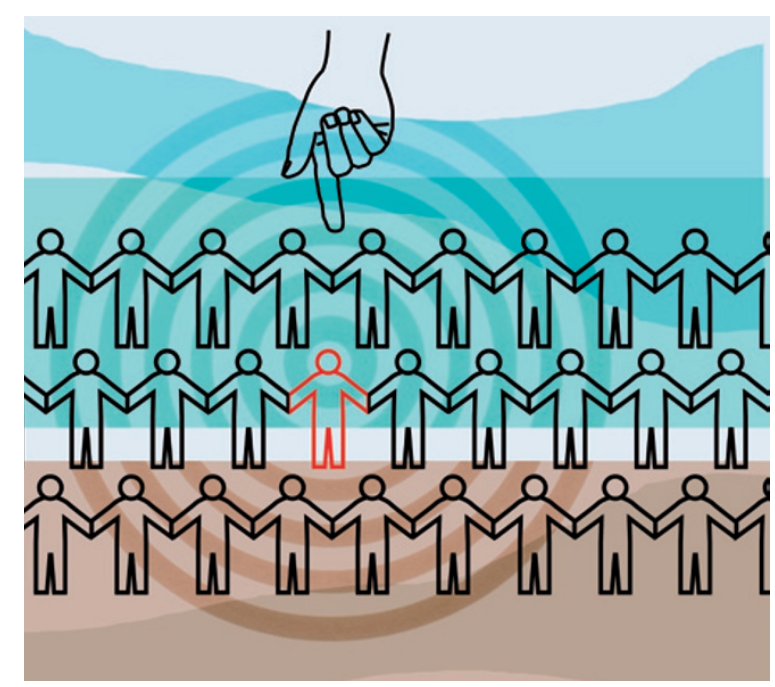

AQP5, which localizes to the apical membrane, in Madin-Darby canine kidney cells. As previously reported, AQP3 was delivered to sites of new cell-cell contact soon after the recruitment of E-cadherin. By contrast, AQP5 remained diffuse over the cell membrane. By fluorescently labelling a small pool of AQP3 that resided in the Golgi, the authors showed that basolateral AQP3 is derived from membrane vesicles that traffic directly from the Golgi to sites of new cell-cell contact.

How might the delivery of proteins from the Golgi to nascent basolateral membrane domains be regulated? Long-range vesicle trafficking is often dependent on microtubules to transport vesicles across the cytoplasm and on membrane tethers that dock vesicles onto the target membrane. These events are followed by SNARE (soluble $N$-ethylmaleimide-sensitive factor attachment-protein receptor)-mediated membrane fusion and integration of vesicle-membrane components into the target membrane. It has been proposed that tethers and SNAREs may form a patch that targets vesicles to the basolateral membrane and is important for protein sorting. In support of this, components of the exocyst complex - a membrane tether - and the SNARE syntaxin-4 localized to sites of cell-cell contact.
Inhibitory antibodies against the exocyst complex, incubation with tetanus toxin (which inactivates some SNAREs) or treatment with a microtubule-depolymerizing agent did not prevent the formation of E-cadherin cell-cell adhesion sites or the delivery of other components of the targeting patch to the membrane. However, in each case, the delivery of AQP3 was blocked. Therefore, microtubules, tethers and SNAREs localize independently to the site of cell-cell adhesion, but coordinate to specify protein targeting to the basolateral membrane.

These findings imply that the formation of cell-cell contacts which is altered in many cancers - is crucial for the establishment of cell polarity. The exocyst complex and syntaxin- 4 are expressed in many polarized cells and might represent a conserved targeting patch by which polarity is established. Further work is required to identify how components of the basolateral membrane targeting patch are delivered to sites of cell-cell contact.

James Pickett

ORIGINAL RESEARCH PAPER Nejsum, L. N. \& Nelson, W. J. A molecular mechanism directly linking E-cadherin adhesion to initiation of epithelial cell surface polarity. J. Cell Biol. 178, 323-335 (2007)

FURTHER READING Rodriguez-Boulan, E. et al. Organization of vesicular trafficking in epithelia. Nature Rev. Mol. Cell Biol. 6, 233-247 (2005) 